\title{
Results from D-T Experiments on TFTR and Implications for Achieving an Ignited Plasma
}

R. J. Hawryluk*

Plasma Physics Laboratory, Princeton University, Princeton, New Jersey, 08543, USA

\begin{abstract}
Progress in the performance of tokamak devices has enabled not only the production of significant bursts of fusion energy from deuterium-tritium plasmas in the Tokamak Fusion Test Reactor (TFTR) and the Joint European Torus (JET) but, more importantly, the initial study of the physics of burning magnetically confined plasmas. As a result of the worldwide research on tokamaks, the scientific and technical issues for achieving an ignited plasma are better understood and the remaining questions more clearly defined. The principal research topics which have been studied on TFTR are transport, magnetohydrodynamic stability, and energetic particle confinement. The integration of separate solutions to problems in each of these research areas has also been of major interest. Although significant advances, such as the reduction of turbulent transport by means of internal transport barriers, identification of the theoretically predicted bootstrap current, and the study of the confinement of energetic fusion alphaparticles have been made, interesting and important scientific and technical issues remain for achieving a magnetic fusion energy reactor. In this paper, the implications of the TFTR experiments for overcoming these remaining issues will be discussed.
\end{abstract}

\footnotetext{
"This paper is coauthored by the TFTR Group. A list of authors in this group can be found in the appendix to this paper.
} 


\section{INTRODUCTION}

Substantial progress has been realized in magnetic fusion science. This has enabled the study of magnetohydrodynamic (MHD) stability and transport in plasmas with reactor-like temperatures and densities and, through the use of deuterium-tritium (D-T) fuel on the Joint European Torus (JET) (JET Team 1992; Gibson and the JET Team 1998) and the Tokamak Fusion Test Reactor (TFTR) (Strachan et al. 1994), the production of significant bursts of fusion energy per pulse. This, in turn, has enabled the study of the confinement and thermalization of alpha particles which are critical to achieving an ignited plasma. With the completion of the TFTR experiments in April 1997 and the worldwide effort on the design of the International Thermonuclear Experimental Reactor (ITER) (Aymar et al. 1997), an assessment of the implications of the TFTR experiments for an ignited plasma is timely.

In assessing the implications of the results from TFTR for achieving an ignited plasma, it is useful to comment on "ignition" and the relationship of the TFTR experiments with ongoing research. Though the concept of an ignited plasma is well defined, even power producing reactors, for control reasons, will most likely be operating "near-ignition" with the goal of minimizing the recirculating power. For this paper, the terms ignition and near-ignition will be used interchangeably. The scientific and technical issues encountered in achieving ignition depend upon the magnetic configuration and the goals of the experimental device. The experiments on TFTR focused on the study of the physics of the reactor core. The results are not only applicable to pulsed tokamaks, with relaxed current profiles, and "advanced performance" tokamaks, with modified current profiles which aim to achieve improved core confinement and MHD stability together with steady-state operation, but also have implications for other toroidal magnetic configurations sharing a common physics basis, in particular, stellarators and spherical torus experiments. This paper begins with a brief description of the TFTR device, followed by a discussion of transport, MHD stability, fusion power production and alpha-particle studies and their implications. A recurring theme throughout is the interrelationship among these topics. This is especially significant in the 
operation of "advanced performance" tokamaks and has implications for other magnetic concepts as well.

In discussing the results from the D-T experiments on TFTR, it is important to note that this work was an integral part of the worldwide effort on tokamak research, and both contributed to and benefited from this effort. This short paper will not attempt to review all of the important contributions from TFTR, nor the critical ancillary technology issues for an ignition device including power and particle exhaust. A review of the results from the D-T experiments on TFTR during the period from December 1993 to December 1996 was recently published by Hawryluk (1998). A historical retrospective of how our understanding evolved from the design of TFTR through the experiments results can be found in Hawryluk et al. (1998).

\section{TFTR DEVICE}

The nearly circular poloidal boundary of the TFTR plasma was set by a limiter on the inboard (high field) side composed of graphite and carbonfiber composite (CFC) tiles mounted on a water-cooled inconel backing plate. A set of poloidal ring limiters composed of carbon fiber composite tiles was used to protect rf launchers on the outboard side which were used to heat the plasma. The limiter could withstand heat outflow from the plasmas of $\sim 30 \mathrm{MW}$ for $1 \mathrm{sec}$. With the configuration shown, metallic influxes from the walls did not contribute significantly to the power balance or the value of the effective ion charge, $Z_{\text {eff }}$. The influx of carbon in high performance discharges was the dominant impurity and resulted in $\mathrm{Z}_{\text {eff }}$ values of $\sim 2$. The principal engineering parameters for TFTR are $B_{T}<6 \mathrm{~T}$, $\mathrm{I}_{\mathrm{p}}<3 \mathrm{MA}, \mathrm{a}=0.9 \mathrm{~m}$, and $\mathrm{R}=2.6 \mathrm{~m}$.

Heating by both neutral beam injection (NBI) and by waves in the ion cyclotron range of frequencies (ICRF) were used on TFTR. The TFTR neutral beam system was composed of four beamlines, each with three positive-ion sources. The ion sources operated either in deuterium or in tritium. The maximum operating voltage was $120 \mathrm{kV}$ and a maximum injected power into a D-T discharge was $40 \mathrm{MW}$. In addition to heating the discharge, the neutral beams were an effective means of fueling the 
discharge. On TFTR, this fueling was varied from all deuterium to all tritium.

The TFTR ICRF system used four antennas to launch magnetosonic waves. Hydrogen and ${ }^{3} \mathrm{He}$ minority, second harmonic tritium, and second harmonic deuterium heating were studied. In addition, fast wave conversion into ion Bernstein waves was used to heat and drive currents in multiple ion species plasmas. During the final experiments on TFTR, a fast wave antenna was replaced with an antenna designed to launch an ion Bernstein wave (Wilson et al. 1998).

Despite the complications introduced by D-T operation, TFTR operated routinely and reliably from the start of D-T operations in November 1993 to the completion of experiments in April 1997. Over 23,000 high power pulses were made, including 1,090 D-T shots. Tritium was used in both the neutral beam and gas injection valves to fuel the plasma. A low inventory tritium purification system was installed and operated in the final phases of the D-T campaign to decrease the shipments of tritium to the facility. More than $0.95 \mathrm{MCi}$ of tritium was processed by the tritium system. The safe and routine operation with tritium on TFTR was an important milestone for the fusion program and provided experience for future ignition machines (Skinner et al. 1998a).

The short term retention of tritium in the plasma facing components and co-deposited layers ranged from $51 \%$ in high performance neutralbeam heated supershot discharges to $>90 \%$ in L-mode discharges. After extensive removal efforts, the long term retention was $\sim 16 \%$ (Skinner et al. 1998b). While this was an operational consideration for TFTR, the implications of these results and modeling studies are that for ITER after the order of one hundred shots the in-vessel tritium inventory will be reached implying the need for highly efficient in-situ cleaning techniques. In addition, in a commercial reactor operating almost continuously, retention fractions $<0.1 \%$ are required (Skinner et al. 1998a).

\section{TRANSPORT}

The attainment of good confinement regimes of operation was a prerequisite for achieving the experimental conditions required for the study of alpha-particle physics in the D-T campaign. The initial high power 
heating experiments on TFTR appeared to confirm the validity and applicability of L-mode scaling in which the energy confinement, $\tau_{\mathrm{E}}$, decreased with auxiliary heating power $\left(\tau_{\mathrm{E}} \propto \mathrm{P}_{\text {aux }}{ }^{-1 / 2}\right)$ and increases with $\mathrm{I}_{\mathrm{p}}$ (Murakami et al. 1986). Strachan et al. (1987) demonstrated that by extensively conditioning the limiters to decrease the influx of deuterium and carbon from the limiters, greatly enhanced confinement times could be obtained in limiter discharges. These enhanced confinement discharges, with $\tau_{\mathrm{E}}$ up to $3 \tau_{\mathrm{E}}{ }^{\text {L-mode }}$, were characterized by beam fueling of the plasma core, peaked density profiles, hot ions with $\mathrm{T}_{\mathrm{i}}(0) / \mathrm{T}_{\mathrm{e}}(0) \sim 2-4$, high edge ion temperature, and strong beam particle fueling. These discharges, commonly referred to as "supershots", have been extensively studied on TFTR. More recently, more effective conditioning techniques involving lithium coating of the limiter were used to further suppress the influx of deuterium and carbon and increase the confinement time (Mansfield et al. 1996). Table I provides a summary of parameters from high performance D$\mathrm{T}$ supershot discharges on TFTR. A consequence of the dependence of the confinement time on the influx of deuterium and carbon was that in the highest power neutral beam heated discharges the confinement time degraded during the heating pulse due to the heat flux on the limiters, which increased the influx of gas from the plasma facing components.

In supershots, $T_{i}(0)>T_{e}(0)$ due to a combination of intense ion heating by neutral beam injection and favorable ion transport. This is advantageous, because a larger fraction of the stored energy is in the reacting ions, which enhances the plasma reactivity. Furthermore, the favorable transport characteristics of this hot-ion regime motivate the development of concepts which enhance the transfer of alpha heating power directly to the ions, such as alpha channeling (Fisch and Herrmann 1998), rather than preferentially to the electrons by classical Coulomb collisions.

The implications of the achievement of the supershot regime were twofold. First, it demonstrated that the empirical scaling laws had limited applicability and could not be relied upon to optimize performance of an existing facility. In supershots, the confinement time remains approximately constant with both neutral beam heating power and the plasma current, whereas in L- and $\mathrm{H}$-mode discharges the confinement is observed to decrease with power and increase with current (Bell et al. 1989). 
Unlike H-mode discharges, the supershot regime continuously evolved from L-mode conditions to enhanced confinement and was not characterized by a bifurcation in transport processes. Thus, the need for exploration and development of an understanding of the underlying physics is and remains important. Second, in discharges with ion temperatures exceeding the predicted optimum for fixed pressure, it was not possible to increase the plasma reactivity by increasing the density. Significant gas puffing increased the edge density and resulted in decreased confinement. The constraint imposed on confinement by the edge conditions is perhaps far more general than usually appreciated. It appears to be manifested in $\mathrm{H}$-mode divertor discharges employing gas puffing resulting in reduction in confinement at densities approaching the Greenwald limit.

As a result of the improved confinement in supershot discharges, the value of $\beta_{\mathrm{p}}$ (which is approximately $\mathrm{p} /\left[\mathrm{B}_{\mathrm{p}}{ }^{2} / 2 \mu_{\mathrm{o}}\right]$ ) became large enough that the bootstrap current predicted by neoclassical theory (Galeev 1970; Bickerton et al. 1971) was significant. In the supershot experiments, the change in surface voltage during beam injection could only be well described by including both the bootstrap current and the beam current which accounted for most of the plasma current in some supershots (Zarnstorff et al. 1988). The availability of co and counter directed NBI in TFTR was crucial to separating the bootstrap and beam driven currents.

The experimental confirmation on a large tokamak of the predicted bootstrap current has had significant ramifications. The bootstrap current is now incorporated in the design of "advanced performance" tokamaks and spherical torus devices seeking to operate in steady-state. In addition, recent theoretical work has indicated that the bootstrap current facilitates the design of certain classes of lower aspect ratio stellarators (Reiman et al. 1998). Also as is discussed below, the bootstrap current can contribute to the destabilization of neoclassical tearing modes.

The achievement of good confinement in TFTR proceeded along two paths: a) exploration using techniques such as wall conditioning and lithium deposition, together with improved limiter alignment and materials to reduce the influx of deuterium and carbon and b) development of increased understanding to provide insight on transport and turbulence suppression. 
Core transport in supershot discharges is substantially reduced compared with L-mode discharges. Local transport studies of supershots indicate that most of the improvement in confinement is associated with the reduction of ion heat conduction (Scott et al. 1990a,b) and ion particle transport (Synakowski et al. 1993). The transport studies also indicate a very different scaling of ion heat, $\chi_{i}$, and momentum, $\chi_{\phi}$, diffusivity with $\mathrm{T}_{\mathrm{i}}$. Meade et al. (1991) observed that $\chi_{\mathrm{i}}$ and $\chi_{\phi}$ decreased with $\mathrm{T}_{\mathrm{i}}$ in the core of supershot discharges whereas they are observed to increase with $T_{i}$ and power in the core of L-mode discharges.

Measurements of the underlying turbulence have been performed and show the following features:

a) Relatively broad spectrum with a peak at $\mathrm{k}_{\perp} \rho_{\mathrm{i}} \sim 0.2$ (Fonck et al. 1993, Mazzucato \& Nazikian, 1993).

b) $\tilde{\mathrm{T}}_{\mathrm{i}} / \mathrm{T}_{\mathrm{i}}>\tilde{\mathrm{n}}_{\mathrm{i}} / \mathrm{n}_{\mathrm{i}}$ indicating that ion dynamics are important (Evensen et al. 1998).

The results from the transport studies, along with the fluctuation measurements, suggest a theoretical model for core transport involving electrostatic modes. Since ion dynamics are important, ion temperature gradient driven modes are candidates. In addition, flow shear, which is believed to be important in the formation of a transport barrier at the edge (Burrell 1997 and references therein), is predicted to suppress turbulence. Furthermore, the electrostatic turbulence calculations indicate that transport could be affected by the current profile. Each of these mechanisms was explored and will be briefly summarized below.

Kotschenreuther et al. (1995) utilizing gyrokinetic linear and gyrofluid nonlinear simulations of the plasma turbulence, reproduced the observed (L-mode) variation of the confinement time and ion and electron temperature profile with plasma current and neutral beam power. The principal transport mechanism in these simulations is low-frequency electrostatic turbulence driven by ion temperature gradients. In the inner half radius, the ion temperature profile is near marginal stability to the mode responsible for the turbulence.

When applied to supershots, the analysis of Kotschenreuther et al. (1995) indicates that the reduction in thermal transport is associated with the suppression of ion-temperature-gradient driven modes due to large values of $T_{i} / T_{e}$, high edge ion temperature and peaked density profiles. 
Although quite successful for L-mode plasmas, the model used by Kotschenreuther et al. (1995) has some important limitations, especially when applied to enhanced confinement regimes. First, the model is not applicable to the edge region (roughly $\mathrm{r} / \mathrm{a}>0.85$ ), and experimental measurements are required to set the boundary conditions. Furthermore, particle transport (and hence heat transport by convection) has not yet been incorporated into the model. Convective heat transport is experimentally inferred to be dominant in the plasma core of supershot discharges. Radial electric field shear was neglected, although more recent versions of the model have taken the toroidal velocity component as an approximation to the radial electric field. Despite these caveats, this model provides a satisfactory description of the performance of L-mode discharges within its domain of applicability and provides insight into the performance of supershots.

Another approach has been pursued by Kinsey and Bateman (1996) using a multi-mode model in which analytic expressions for the transport associated with various models are used to describe the dependence of plasma energy and particle transport. In this approach, the numerical factor setting the transport level for each model is obtained by fitting the experimental data and evaluating the overall fit to the data. The edge conditions are not taken from experimental data; however, the influx of neutrals from the edge is adjusted to obtain agreement with the measured density. The transport is predicted to be governed by electrostatic drift-wave turbulence and ballooning modes. The agreement between theory and experiment is satisfactory for a broad range of conditions. Further tests of this model employing both a wider range of data, as well as response to plasma perturbations, are required to evaluate the range of validity of the multi-mode model.

The scaling of confinement time with isotopic mass is important not only for projecting the performance of an ignition device, but also as a test of different transport models. It was immediately apparent in the initial TFTR D-T experiments that the global energy confinement in supershots is significantly better (20\%) in D-T plasmas than in comparable D plasmas (Hawryluk et al. 1994; Scott et al. 1995). This favorable result was also evident in high-l $l_{i}$ plasmas with relatively peaked current profiles including those with H-mode transitions. In L-mode D-T plasmas (Scott et al. 1997) on 
TFTR, the global and thermal plasma energy confinement scale at least as favorably with average ion mass as the $\tau_{\mathrm{E}} \propto<\mathrm{A}_{\mathrm{HYD}}>^{0.5}$ dependence embodied in the ITER-89 L-Mode scaling. Most transport theories involving small scale turbulence scale as gyro-Bohm i.e. $\chi \propto \chi_{\mathrm{B}} \rho^{*}$ where $\chi_{\mathrm{B}}=\mathrm{cT}^{\mathrm{T}} \mathrm{eB} \mathrm{B}_{\mathrm{T}}, \rho^{*} \equiv \rho / \mathrm{a}$ and $\rho$ is the ion Larmor radius and would therefore predict an unfavorable scaling with ion mass, in disagreement with the TFTR observations and many other previous experiments conducted in hydrogen and deuterium (Bessenrodt-Weberpals et al. 1993).

As in the case of H-mode transport barrier formation (Burrell 1997), another mechanism which can be important in both L-mode and supershot discharges is flow shear stabilization of the turbulence. The favorable ion thermal confinement trends of supershot discharges can be reproduced by self consistently including the effects of a neoclassical radial electric field, using the linear parts of the model of Kotschenreuther et al. (1995) to describe the stability of ion-temperature-gradient driven modes (Ernst 1997; Ernst et al. 1998a,b). Analysis of a large number of high power supershot experiments indicates that by including flow shear stabilization in this way, it is possible to reproduce the dependence of $\chi_{i}$ on heating power, recycling, density peakedness, toroidal rotation, and isotopic mass. The large nonmonotonic toroidal velocity profiles of the impurity ions are consistently explained by the same neoclassical calculation which provides the radial electric field. However, recent direct measurements of the carbon poloidal rotation velocity indicate a discrepancy with the calculated values which remains to be understood. The initial analysis indicates that the measured poloidal flow velocity (and hence the radial electric field) appears to be correlated with changes in confinement (Bell et al. 1998). In addition to the results from the supershot experiments, Scott et al.(1997) have shown that the inclusion of shear flow effects in the ion-temperature gradient turbulence model also reproduces the observed isotopic effect in L-mode plasma. Beam injection can be used to apply a torque to the plasma to induce plasma rotation. In L-mode discharges, the confinement time was observed to depend on the direction of beam injection with respect to the plasma current. This is another indication that flow shear is important, and raises the question of the range of validity of empirical scaling relations which do not take into account the externally induced flow shear in the plasma. 
The emerging theoretical transport models which are still being benchmarked on TFTR data have important ramifications for ignition experiments due to the dependence on flow shear, edge density, ratio of $\mathrm{T}_{\mathrm{i}}(0) / \mathrm{T}_{\mathrm{e}}(0)$ and isotopic composition.

Experimental work on a number of devices indicated a reduction of core transport associated with the formation of regions of reversed shear. These initial experimental observations were further supported by the theoretical work of Kessel et al. (1994) who predicted that plasma transport could be reduced in discharges with reversed magnetic shear. The recent development of operational techniques to reliably create this magnetic configuration, coupled with new diagnostics to measure the pitch of the magnetic field on TFTR (Levinton et al. 1995), DIII-D (Strait et al. 1995) and JT-60U (Fujita et al., 1997) have resulted in exciting new results.

To create a reversed magnetic shear configuration, the plasma is typically started at full size and the current is increased rapidly. Since the current diffusion time is slower than the rise time of the total plasma current, the current density profile, $j(r)$, is hollow during and, for some time after the increase.

The confinement characteristics of reversed shear shots on TFTR in the high power heating phase resemble supershots with the same machine parameters. In particular, the global confinement time is enhanced relative to L-mode scaling and convection is important in the core power balance. However, above a neutral beam power threshold (which depends on machine conditioning, the q-profile and the magnetic field) the core transport changes abruptly at $0.2-0.3 \mathrm{sec}$ into the main heating phase within the region of reversed shear. In TFTR discharges, the effect is most clearly seen on the central density evolution which can rise by more than a factor of 2 in $0.3 \mathrm{sec}$ (see Fig. 1). Since the density outside the reversed shear region changed little, the density profile following the transition became very peaked, reaching values of $n_{e}(0) /<n_{e}>\sim 5$. This state of improved confinement in the core of reversed shear plasmas is known as enhanced reverse shear (ERS) in TFTR.

As shown in Fig. 2, at the transition the inferred electron particle diffusivity in the region of the steepest density gradient drops by a factor of 
10-50 to near neoclassical levels, while the ion thermal diffusivity falls to levels well below predictions from conventional neoclassical theory (Levinton et al. 1995). Similar improvements in the ion momentum diffusivity have also been observed. The region of steepest pressure gradients and where the transport coefficients drop is where a transport barrier is assumed to form. Recent calculations by Lin et al. (1997) indicate that a more comprehensive analysis of neoclassical transport, which considers orbit dimensions comparable with pressure scale lengths, is in better agreement with the data in the enhanced confinement regime. Inasmuch as neoclassical transport is usually thought to be the minimum transport possible, these results represent a dramatic improvement in confinement.

The ERS transition has been correlated with the suppression of turbulence by the $\mathbf{E x B}$ shear flow; that is when the shearing rate $\gamma_{\mathrm{s}} \equiv$ $\left|\left(\mathrm{RB}_{\mathrm{p}} / \mathrm{B}\right) \mathrm{d} / \mathrm{dr}\left(\mathrm{E}_{\mathrm{r}} / \mathrm{RB}_{\mathrm{p}}\right)\right|$ exceeds the plasma turbulence decorrelation rate, which is estimated to be about the linear growth rate of the mode responsible for the turbulence. After the ERS transition, the fluctuation level in the core is dramatically suppressed according to reflectometer measurements (Mazzucato et al. 1996). Experiments on TFTR indicate that ExB shear is necessary to maintain an internal barrier and that the gradient in the Shafranov shift (which is increased in reversed shear discharges) is not sufficient to maintain the barrier (Synakowski et al. 1997). More generally, while reverse shear has facilitated the achievement of transport barriers on TFTR, it is not a requirement for the attainment of transport barriers. In particular, experiments on TFTR and other devices have produced internal transport barriers with weak positive or negative shear. In the final series of TFTR experiments, poloidal rotation profiles have been measured using a recently developed diagnostic to study the onset of the internal transport barrier (Bell, Levinton, Batha, Synakowski \& Zarnstorff 1998). This diagnostic, together with Stark Effect measurements, showed that in many discharges studied which undergo a transition prior to the formation of an internal transport barrier, a highly localized and transient excursion in the poloidal flow velocity and radial electric field occurred (Levinton et al. 1998). The induced poloidal flow is much larger 
than predicted by neoclassical theory. Presently, the role of this excursion in the dynamics of the barrier formation is being investigated theoretically.

Though the agreement between theory and experiment is promising, the present models do not adequately describe the dependence of the power threshold of the ERS regime on the toroidal field and neutral beam induced rotation. Also, it is unclear whether the higher power threshold for ERS discharges in T versus D can be readily reconciled with simple ExB mechanisms (Scott et al. 1997). Another topic requiring further investigation is the apparent decoupling of the electron and ion heat diffusivity. Recent analysis indicates that the electron heat diffusivity appears to decrease modestly within the transport barrier but increases in the core (Bell et al. 1998). These results suggest that more than one mechanism is important in core transport of enhanced reverse shear discharges. Despite the need for additional experimental and theoretical work, the emerging physics models of electrostatic turbulence and stabilization by flow shear are providing a valuable tool to describe the underlying physics and motivate new experiments to suppress turbulence.

For an ignition experiment, the question is how to attain and control an internal transport barrier. If the barrier is a consequence of a steep pressure and density gradient driving poloidal flows (as indicated in the TFTR experiments) then this effect would be smaller in ignition devices with smaller values of $\rho^{*}$ or less central fueling. If the barrier is a consequence of the external torque applied using neutral beam injection and the resulting induced toroidal rotation (as indicated in the DIII-D experiments), then the power required to obtain and sustain a comparable Mach number for an ignition device could be significant. One approach investigated during the final experiments on TFTR was to induce flow shear by the application of ion Bernstein waves. This technique had been used on Princeton Beta Experiment-Modification (PBX-M) by Ono (1993) to generate an internal transport barrier. However, measurements of the induced flow velocities were not available in those experiments. On TFTR, the poloidal flow measurements demonstrated that ion Bernstein waves can induce poloidal flow; however, with the limited available time insufficient power was coupled to the core to evaluate whether suppression of transport can be achieved (Wilson et al. 1998). Efficient techniques to 
control the formation of transport barriers in ignited plasma remain to be developed.

\section{MHD STABILITY}

Two dimensional MHD codes are used to predict the ideal MHD stability limits. Troyon et al. (1984) and Sykes et al. (1983), for "optimized" pressure and peaked current profiles, characterized their results in terms of the parameter $\beta_{\mathrm{N}} \equiv \beta_{\mathrm{T}} /\left(\mathrm{I}_{\mathrm{p}} / \mathrm{aB}_{\mathrm{T}}\right)$. Troyon et al. (1984) showed that the pressure limit associated with low-n kink modes corresponds to $\beta_{\mathrm{N}}<2.8$, whereas, Sykes et al. (1983) showed that for ballooning modes $\beta_{\mathrm{N}}<4.4$. The predictions of ideal MHD have important ramifications, for they imply that for a given facility with sufficient auxiliary heating and plasma confinement, the maximum fusion power is limited by the available plasma current and toroidal field. An implicit consequence is that, since the total pressure governs stability and only the ion pressure contributes to fusion reactivity, operation in regimes in which $\beta_{\mathrm{i}}>\beta_{\mathrm{e}}$ increases fusion power performance. This motivates operation in the hot-ion regime.

In high-performance supershot discharges, the sawtooth instability is typically stabilized even when the central safety factor, $q(0)$, is less than one. Levinton et al. (1994) showed that when the pressure and density profiles are sufficiently peaked compared with the shear in the q-profile, stabilization occurs as predicted by a two-fluid MHD model. The stabilization of sawteeth has both practical, as well as theoretical implications. Since the competing effects of redistribution of particles and energy by the sawteeth are not present in high performance discharges, this has facilitated transport studies and the identification of alpha heating. Theoretically, the observation of $q(0)<1$ has significant consequences for the predicted pressure limits, as is discussed below.

Experimentally, there are significant limitations to the range of pressure and current profile which can be produced while achieving good confinement and the control of the evolution of these profiles. Ideal MHD theory predicts that $\beta_{\mathrm{N}}$ depends on the peaking of the plasma and current profile for discharges with monatomic q-profiles and $q(0) \geq 1$. As noted earlier, the supershot regime is characterized by peaked density and pressure profiles. The discharge with the highest confinement $(\sim 0.3 \mathrm{sec})$ 
was achieved by means of extensive wall conditioning and Li deposition to control the influx of deuterium and carbon into the discharge. This discharge disrupted at a relatively low value of $\beta_{\mathrm{N}}=1.5$, whereas the highest fusion power discharge with broader pressure profiles achieved $\beta_{\mathrm{N}}=1.8$. Ideal MHD theory predicts that the maximum value of $\beta_{\mathrm{N}}$ would decrease with pressure profile peakedness as qualitatively observed. However, the parameter $\beta_{\mathrm{N}}{ }^{*}=\left(\left\langle<\mathrm{p}>>/\left(\mathrm{I}_{\mathrm{p}} / \mathrm{aB}_{\mathrm{t}}\right)\right.\right.$ where $\left.\langle<\mathrm{p}\rangle>=\left[\int \mathrm{p}^{2} \mathrm{dV} / \int \mathrm{dV}\right]^{0.5}\right)$ which is more relevant for fusion power production is predicted to be a weaker function of pressure profile peaking for circular plasma cross-section tokamaks such as TFTR. The ideal MHD theory predicts that the stability limit should increase with the peaking of the current profile, or alternatively, the internal inductance. This has been successfully demonstrated by ramping the current down or by modifying the plasma initiation to peak the current profile. By means of current rampdown, $\varepsilon \beta_{p}<1.6$, where $\varepsilon=R / a$, has been achieved with a separatrix inside the vessel and $\beta_{\mathrm{N}}<4.7$ has been achieved (Sabbagh et al. 1995). These results demonstrate that ideal MHD calculations provide insight into approaches to optimize the performance of the machine. Quantitatively, there is one outstanding issue. In many TFTR discharges $q(0)<1$. For such profiles the codes predict a vigorous internal $\mathrm{n}=1, \mathrm{~m}=1$ mode which is not observed. Instead this mode is stable or saturated at a low level. To obtain agreement with experiment, the codes assume $q(0) \geq 1$; however, a comprehensive treatment of this mode is still needed.

The TFTR results highlight the importance of controlling the pressure and current profile to optimize the performance of an ignited plasma, and the requirements for transport predictions of the pressure and current profiles for a device operating near the ideal limits. While the above considerations give an overview of the important trends regarding the pressure limits on TFTR, detailed measurements and comparison with theoretical work provide greater insight into the underlying dynamics. Two different mechanisms appear to be responsible for the $\beta$-limit in supershot discharges on TFTR: neoclassical tearing modes and intermediate-n ballooning instabilities coupled to a lower-n kink mode. The MHD resistive tearing mode theory was extended to the long mean-free-path regime by incorporating neoclassical effects including the bootstrap current. The onset of tearing modes was found to degrade the confinement, the plasma 
stored energy, and the neutron emission in the discharges, but did not typically result in an abrupt termination of the discharge (Chang et al. 1995). These modes appear spontaneously, have low frequencies $(<50 \mathrm{kHz})$, and low poloidal (m) and toroidal (n) wave numbers with $\mathrm{m} / \mathrm{n}=3 / 2,4 / 3$, and $5 / 4$. In contrast with the results on smaller devices, the $2 / 1$ mode is rarely observed, but if it occurs it is particularly detrimental to confinement. The nonlinear evolution of these modes agrees well with the predictions of neoclassical pressure-gradient-driven tearing mode theory. The predicted evolution of the island width is found to be in reasonable agreement with measurements. Though the present theory predicts several important trends in the data, it does not predict a threshold island width, and therefore, which modes should grow and when. An important implication of the theory is that localized current drive (for instance by electron cyclotron waves) can be used to stabilize the modes. Experiments are beginning on a number of facilities to test this prediction.

In discharges on TFTR with high toroidal field and plasma current, the neoclassical tearing modes were not the most important limitation. In these discharges, the maximum stored plasma energy was limited by the onset of a rapidly growing intermediate- $n$ ballooning instability $(n=10-20)$ coupled to a low-n ideal kink mode (Nagayama et al. 1993; Fredrickson et al. 1996). The distortions to the plasma caused by the large low-n ideal mode appear to push the plasma over the ballooning mode stability boundary. The ballooning-mode precursor to the disruption was studied by electron cyclotron emission measurements from two grating polychromators separated toroidally by $126^{\circ}$ to determine the electron temperature. From these measurements, it is possible to deduce that the ballooning mode is both toroidally and poloidally localized on the outboard midplane. Park et al.(1995), using a three-dimensional nonlinear MHD code, successfully modeled the observed electron temperature fluctuations. In this simulation, a saturated low $\mathrm{n}$ mode is assumed to be present. These simulations indicate that the high-n mode becomes even more localized, producing a strong pressure bulge that destroys the flux surfaces, resulting in a thermal quench. This instability limits the maximum fusion power achieved in supershots on TFTR and can result in a plasma disruption.

The stability of reversed shear discharges provide a valuable test bed for checking our understanding of MHD stability since $q_{\min }>1$ and the 
complications associated with the stability of the $m=1, n=1$ mode are avoided. In addition, they have the potential for increased performance (Kessel et al. 1994). In the region of reversed shear, MHD activity is absent in ERS discharges as measured by the four channel reflectometer, suggesting that, as predicted by theory, reversed shear plasmas may indeed have greater local MHD stability. The local pressure gradient in flux coordinates in enhanced reversed shear discharges on TFTR is larger, by a factor of 3-5, than in typical supershots with monotonic q profiles, which very often have low-n MHD modes in the core. However, as the transport barrier moves into the weak or positive shear region and as the radius of the minimum value of $\mathrm{q}\left(\mathrm{r}_{\mathrm{min}}\right)$ moves to the core region, a rapidly growing $\mathrm{n}=1$, ideal-like MHD instability is observed to disrupt the plasma. The maximum pressure appears to be limited in this region by the ideal infernal mode. Comparison of the structure of the observed and calculated mode is in good agreement, and the threshold is in reasonable agreement (Manickam et al. 1997).

The MHD stability is governed by the evolution of the current profile and the development of an internal transport barrier with sharp pressure gradients. The implications of the TFTR experiments are that, to take advantage of the promise of the reversed shear regime, the development of efficient techniques to control the current and pressure profile are required. In a reactor in which self heating and local transport coefficients will determine the pressure profile, it may be necessary to develop techniques to control transport or transport barrier formation to obtain good MHD stability.

\section{Fusion Power Production}

The achievement of good confinement and high pressure were prerequisites for proceeding with D-T experiments to study alpha-particle physics and demonstrate fusion power production (Hawryluk et al. 1994; Strachan et al. 1994). The discharge with the highest fusion power on TFTR is shown in Fig. 3. The decrease in fusion power is triggered by MHD instabilities (a minor disruption) followed by a carbon bloom (sudden influx of carbon into the plasma from the limiter). The fusion power densities

achieved in the center of high-performance TFTR supershots, up to 2.8 MW 
$\mathrm{m}^{-3}$, are comparable to, or greater than, those expected in ITER. The value of $\mathrm{P}_{\text {fus }} / \mathrm{P}_{\text {aux }}$ reached 0.27 and the total fusion yield from a single plasma pulse on TFTR reached 7.6 MJ.

Simulations of the neutron production on TFTR have been performed using the TRANSP data analysis code (Budny 1994; Ongena, Evrard \& McCune 1998). This code uses the measured electron density and temperature profiles, ion temperature profile, and visible bremsstrahlung measurements in conjunction with other measurements and engineering data, such as the beam heating power and source divergence, to calculate the neutron source rate from thermal, beam-thermal, and beam-beam reactions. Monte Carlo techniques are used to compute the deposition of the neutral beams, and the distribution of the beam ions and fusion ions, such as alpha particles. The beam ions and fusion products are assumed to slow down classically. The overall agreement for both the time dependence of the D-T neutron emission and the neutron emissivity profile is well described by the TRANSP code for TFTR supershot discharges. Though the agreement is good in supershot discharges, detailed comparisons of the predicted neutron emission with experiment in reverse shear discharges

have identified some discrepancies. Initial analysis indicates that only part of the discrepancy between the TRANSP prediction and measurements is due to stochastic ripple diffusion.

\section{AlPha-Particle Physics}

The alpha-particle confinement experiments on TFTR have been reviewed by Zweben et al. (1997) and by Hawryluk (1998) and are summarized in this section. These results are discussed in terms of the conventional description of alpha particles typically used in calculating the performance of new devices. In the conventional approach, the confinement of alpha particles is described in terms of three mechanisms: neoclassical processes (single particle orbit effects); the transfer of power by Coulomb collisions; and the enhanced transport of the alpha ash from the core by turbulent processes.

Measurements of the confined alpha particles, using the charge exchange recombination measurements, as well as the loss measurements, indicate that in MHD quiescent discharges the radial 
diffusion coefficient for alpha particles is indeed very small, and up to 10 times smaller than that of the thermal ions as shown in Fig. 4 (McKee et al. 1997). A possible explanation is that "orbit averaging" effects, which occur when the ion banana width is large compared to the turbulence correlation length, reduce the effect of the underlying background turbulence (Mynick \& Duvall 1989; Myra et al. 1993; Manfredi \& Dendy 1996). The size of the reduction in alpha transport in larger devices may depend on the scaling of the size of the turbulence correlation length with machine size. The good confinement of beam ions in present machines (Heidbrink \& Sadler 1994) with smaller orbits than those of fusion products supports the hypothesis that the alpha particles should be well confined in larger devices.

The good agreement between the measured alpha-particle energy spectrum and theory indicates that the transfer of power is well described by Coulomb collisions and orbit effects (Fisher et al. 1995; McKee et al. 1995; McKee et al. 1997; Medley et al. 1998). These results are also consistent with the observations of beam-ion thermalization on TFTR in D and D-T plasmas as well as many other devices (Heidbrink \& Sadler 1994).

Another confirmation of the conventional model is the measurement of helium ash accumulation. The modeled helium ash time evolution indicates that the alpha-particle slowing-down calculations and transport assumptions in supershot discharges for the ash are consistent with measurements. Best agreement between the modeling and measurements is obtained using the measured radial particle transport and wall recycling coefficients for the thermal helium. When the slowing-down rate is varied by a factor of 2 , the data falls within the predicted evolution of the ash density. The time behavior is inconsistent with large anomalous energetic alpha particle loss (Synakowski et al. 1995). The data do not exclude the possibility of modest $(20 \%-30 \%)$ losses due, for example, to unobserved MHD instabilities.

In the highest performance D-T discharges produced on TFTR, the alpha-particle heating is a relatively small fraction of the total power heating the plasma, making its detection difficult. Nevertheless, the electron temperature rise in TFTR D-T shots during beam injection is greater than in D-only or T-only shots (Hawryluk et al. 1994; Budny et al. 1994; Taylor et al. 1996). Analysis indicates that the change in electron temperature requires including alpha-heating and isotope effects. When 
the database is constrained to take into account the change in electron temperature associated with confinement, the residual change has been determined to be in reasonable agreement with the predicted alpha heating as shown in Fig. 5. Very recently, Thomas et al. (1998) reported larger increases in electron temperature $(1.3 \mathrm{keV})$ in JET alpha-particle heating experiments to also be in accord with predictions.

Further experiments with a higher ratio of alpha heating to heating power will be required to evaluate the efficiency of alpha heating due to its importance for reactor design. Though a great deal has already been learned about both the confined and escaping alpha particles, it must be kept in mind that the present measurements sample only part of the distribution function (Zweben et al., 1997) and that the observed heating is small and complicated by changes in confinement. Thus, direct measurements of the alpha-particle heating effectiveness are important to check the validity of the computational modeling. This would be a major contribution of an ignited plasma experiment.

MHD activity is found to affect the confinement of alpha particles. For example, sawteeth can radially redistribute alpha particles. Substantial loss (up to 20\%) to the walls occurs during disruptions. Enhanced loss has been observed during the occurrence of neoclassical tearing modes and kinetic ballooning modes. Most of the MHD- and ICRFinduced alpha-particle loss observed on TFTR occurs at the passingtrapped particle boundary, at which point alpha particles in the core of TFTR can go onto loss orbits. This effect should be smaller in future larger devices such as ITER with a smaller first-orbit loss region; however, the analogous ripple loss region could be sensitive to the wave-induced internal diffusion of alpha particles (Zweben at al. 1997).

Energetic particles such as alphas can also destabilize (or in some cases stabilize) MHD modes (Heidbrink \& Sadler 1994). This is a potentially important topic because of the coupling between alpha-heating and MHD stability in a reactor, as well as the loss of alpha particles to first wall components caused by the induced collective instabilities. In this paper, the effect of alpha particles on the stability of toroidal Alfvén eigenmodes (TAE) will be discussed.

Toroidal Alfvén eigenmodes have been shown to exist with discrete frequencies inside the shear Alfvén continuum gaps created by toroidal 
coupling of different poloidal harmonics. These modes were predicted to be driven unstable by energetic particles through wave-particle resonances by tapping the free energy associated with the energetic alpha pressure gradient (Cheng, Fu \& Van Dam 1989). Since this initial theoretical work, a large theoretical literature has developed which includes additional important effects, both in the analysis of the instability criteria, as well as in the nonlinear saturation mechanisms. Experiments on TFTR and DIII-D have shown that the toroidal Alfvén eigenmode could be destabilized by the energetic ion populations created either by neutral beam injection or ICRF heating (Heidbrink \& Sadler, 1994). These instabilities can be sufficiently strong to eject a large fraction of the fast particles (Darrow 1997) and damage the first wall.

The initial D-T experiments on TFTR in supershot and L-mode discharges, however, showed no signs of alpha-driven instability in the TAE frequency range, and the alpha-particle loss rate remained a constant fraction of the alpha production rate as the alpha pressure increased, suggesting that deleterious collective alpha instabilities were not being excited. Theory has since shown that, although TFTR achieves levels of the alpha-particle driving terms nearly comparable to those of a reactor, the damping of the mode in TFTR is generally stronger than the alpha-particle drive. Subsequent theoretical calculations have shown that the predicted alpha-driven TAE threshold is sensitive to the q-profile and beam damping (Spong, Hedrick \& Carreras 1995; Fu et al. 1996; 1997; Berk et al. 1997). The dependence on the q-profile is potentially important in advanced tokamak configurations in which the current profile is modified to achieve higher stability. In experiments with weak magnetic shear on TFTR, TAE driven by energetic alpha particles have been observed in D-T plasmas (Chang et al. 1997; Nazikian et al. 1997). These modes are observed only after the end of the high power heating phase when the beam ion density and plasma pressure are decaying more rapidly than the alpha pressure as shown in Fig. 6. The fusion power threshold is $\sim 1.0 \mathrm{MW}$ with $\beta_{\alpha}(0) \sim 10^{-4}$ for $\mathrm{q}(0) \simeq$ 2.4. This threshold is much lower than the value of $\beta_{\alpha}(0)$ obtained in highpowered supershot discharges with $\mathrm{q}(0) \leq 1$ and monotonically increasing $\mathrm{q}$ profile, which were observed to be stable. The onset of mode activity is generally consistent with NOVA-K code linear stability calculations, though the poloidal mode structure remains to be clarified (Chang et al. 
1997). The mode amplitude increases with increasing fusion power. The TAE mode with weak shear is potentially important in both advanced tokamak configurations, in which the current profile is modified to achieve higher pressure, and in certain stellarator designs.

While the TFTR results have clarified the threshold criteria for alpha driven TAE instability, the amplitude of this mode was very small and no loss of alpha particles was detected. This is consistent with the general arguments presented by White (1995) which indicate that little radial transport would occur due to a weak single mode. In ignition machines, higher values of the mode number may be excited, and overlapping modes may result in stochastic diffusion and perhaps increased transport.

Several concepts have been proposed to use alpha particle-wave interactions to more effectively utilize the alpha particles in a reactor (Fisch and Rax 1992; Mynick and Pomphrey 1994; Hsu et al., 1994; Herrmann and Fisch 1997; Fisch and Herrmann 1998). By coupling the alpha particle energy to a plasma wave which then deposits its energy in the plasma, it is theoretically possible to: 1) transfer the energy of alpha particles preferentially to the ions and thereby increase the plasma reactivity and reduce the alpha pressure; 2) radially redistribute the alpha particles for alpha-ash control; 3) control the alpha-heating profile which may enable pressure profile control in a tokamak; 4) control the alpha-pressure profile which may further reduce the drive for adverse alpha-particle instabilities; 5) transfer momentum to the electrons for current drive. While it may not be possible to achieve all of these objectives simultaneously, this concept potentially offers additional flexibility which may be valuable to enhance the performance of a reactor.

Numerical simulations indicate that for a reactor-size plasma, the simultaneous excitation of both TAE and mode-converted IBW can be used to extract more than half the energy from the alpha particles (Herrmann \& Fisch 1997). Experiments on TFTR focused on understanding the physics of energetic particle interaction with ion Bernstein waves. The experimental results (Darrow et al. 1996; Fisch and Herrmann 1998) to date support the underlying assumptions of wave propagation in the models which predict the feasibility of alpha channeling. Recent analysis of the TFTR experiments by Herrmann (1998) suggests that the collisionless energetic 
particle diffusion rate due to interaction with ion Bernstein waves is larger than previously estimated which may decrease the auxiliary power required for achieving alpha channeling (Fisch and Herrmann 1998). Much more experimental and theoretical work is required to establish this novel approach. In particular, one major element which remains is to establish the transfer of power from the alpha particles to the rf wave.

In the theoretical work by Fisch and Rax (1992) stochastic waveparticle interactions diffuse the alpha-particle orbits in a preferred direction by the appropriate choice of wave numbers and wave direction. Another approach, relying upon frequency sweeping of the wave, has been proposed by Mynick and Pomphrey (1994) and Hsu et al. (1994). This mechanism can move particles with a selected parallel velocity in a nondiffusive fashion from one specified radius to another. This technique may be used to control the density of $\mathrm{He}$ ash and also requires experimental tests to evaluate the efficiency of removing a significant part of the distribution function.

\section{IMPLiCATIONS For IGNition DEVICES}

In present D-T experiments, the pressure profile, the current profile and the rotation profile are largely determined by the application of auxiliary heating, inductive and non-inductive current drive and the applied torque whereas alpha heating has a small effect on the pressure profile. While this external control of profiles has facilitated the study of the underlying transport and plasma stability in present day experiments, it can not be representative of conditions in an ignited plasma.

In an ignition device, the operating temperature cannot be simply controlled because the heating power cannot be directly controlled.

The problem becomes more complex when one considers the constraints on an economically attractive device operating in steady state. An economically attractive reactor, in addition to minimizing the capital cost for construction, needs to minimize the recirculating power including the power for controlling the plasma. The self-generated internal processes become dominant for $\mathrm{P}_{\alpha} \sim \mathrm{P}_{\text {loss }}>>\mathrm{P}_{\text {aux }}$ as illustrated in Fig. 7. In an ignited plasma, the pressure profile will determine the alpha heating profile, the bootstrap current, and a significant component of the radial electric field. 
In addition, the interaction among the pressure, current, and rotation profiles can become much greater, especially in steady-state operating regimes with higher values of $\beta$. In a steady-state tokamak, the fraction of bootstrap current must be large, otherwise the power required for external current drive using present techniques would be too large (Ozeki et al., 1993). An alternative approach is to design a pulsed device similar in concept to present machines; however, as discussed by Bathke et al. (1994), this may be less economically attractive. The bootstrap current modifies the current profile, which in turn modifies the radial electric field shear due to the change in the poloidal magnetic field. The radial electric field shear may decrease the radial transport, increasing the plasma pressure, which in turn increases the alpha-heating. The rotation profile and, hence, the radial electric field, would be largely defined by the plasma pressure gradients because to drive significant plasma flows, the power required would be too large using neutral beam injection. (This also motivates the development of rf techniques for flow shear generation as discussed above.) Hence, it is not certain that a self-consistent stable operating point exists, for the processes are highly nonlinear and require a detailed knowledge of the underlying transport mechanisms beyond what has so far been demonstrated. Furthermore, the problem becomes even more complex when MHD stability, which can interact with the alpha particles and is sensitive to the current and pressure profiles, is taken into account. In future ignited machines, the role of the external heating and current drive, as well as applied torque, will be to control and modify the plasma either through the alpha particles, such as by alpha channeling, or by altering the underlying transport mechanisms or by localized current drive. Controlling the operating point of a burning plasma with a minimum of auxiliary power is both a major scientific and technological challenge.

These considerations have significant ramifications for the mission and goals of an ignition experiment. The triple product $\mathrm{n}_{\mathrm{Hyd}} \mathrm{T}_{\mathrm{i}} \tau_{\mathrm{E}}$, which provides an estimate for the parameters required for ignition, can be rewritten $\beta \tau_{\mathrm{E}} \mathrm{B}^{2}$ for $\beta_{\mathrm{Hyd}} \sim \beta_{\mathrm{e}}$. Subject to technical constraints such as stresses on the magnets and heat loads on plasma facing components, it should be possible to achieve ignition at relatively lower values of $\beta$ by operating at higher values of magnetic field and/or larger values of confinement time. At reduced values of $\beta$, it will be possible to study the 
effectiveness of alpha-particle heating and decrease the complications associated with increased coupling between transport and MHD stability. However, for an economical reactor, the control of a burning plasma for long durations with relaxed profiles and at relatively high $\beta$ is an important scientific and technical issue. This motivates placing a premium on being able to address some of these issues in any ignition device. The conundrum is that while in the future the advanced performance tokamak regimes with both good confinement and high $\beta$ may result in a more economical reactor the experimental database required for the design of an advanced tokamak ignition device is only now coming into existence. Thus, the design of a tokamak ignition device today can not take advantage of the potential cost savings associated with the advanced performance regimes.

Though the tokamak has demonstrated parameters much closer to ignition than any other magnetic configuration, other configurations have potential advantages to address the control issue. For instance, a stellarator does not require external current drive and can be optimized to either have the equilibrium dependent on the bootstrap current or not. Furthermore, present results indicate that stellarator discharges do not undergo major disruptions; however, additional experiments near the pressure limits are required to fully assess the plasma response. The higher predicted values of $\beta$ in the spherical torus, which have been achieved on the START experiments (Gryaznevich et al. 1998), could enable operation away from the nominal pressure limits and decrease the requirements on the control systems. In addition, spherical torus reactors would operate at high $\rho^{*}$ and have significant radial electric field gradients from the pressure gradient.

Though the control of a burning plasma is a significant issue, the evolving understanding of the underlying transport mechanisms, MHD stability and wave-particle interactions in both $\mathrm{D}$ and D-T experiments is providing the tools necessary for this task. Advanced computational techniques are being developed and benchmarked on present experiments both to evaluate these issues and develop new concepts to control a burning plasma.

\section{SUMMARY}


Substantial progress has been made in fusion technology, plasma diagnostics, experiments, and theory which has increased our understanding of the underlying physics, and enabled the study of the physics under conditions similar to that in the core of a reactor.

In the area of transport, enhanced confinement regimes with and without transport barriers at the edge indicate opportunities for further improvement in performance and have been a critical element in the achievement of reactor-grade plasma on TFTR. Experimentally, the edge conditions have been important in the achievement of enhanced confinement regimes and imposed constraints on optimizing the plasma reactivity. Several of the underlying mechanisms associated with transport suppression and transport barrier formation are emerging both theoretically and experimentally. Theoretical predictions are increasingly being used to motivate experiments to improve performance. The resulting experiments benchmark the theory and associated codes. However, further work is needed to establish a quantitative predictive capability. MHD instabilities due to pressure driven modes were a major consideration in the experimental program. MHD theory was increasingly used to guide experimental design and operation. The observation of a strong interplay between the $\mathrm{n}=1$ kink and ballooning modes demonstrates the need for three dimensional MHD codes to clarify the disruption mechanism. The observation of neoclassical tearing modes has shown the importance of the inclusion of kinetic effects appropriate to the long-mean-free-path regime associated with the core of a tokamak.

For the first time, a comprehensive study of alpha-particle physics was performed. In MHD quiescent discharges, the alpha-particle behavior is consistent with the conventional model of neoclassical transport and transfer of energy by Coulomb collisions. The first indications of alphaheating were obtained. However, MHD instabilities can and do affect the radial redistribution and loss of alpha particles. Alpha particles have also destabilized the toroidal Alfvén Eigenmodes in weak shear discharges, which was an important test of energetic particle interaction with MHD waves.

It is clear major progress has been achieved not only in higher values of $\mathrm{nT} \tau$ and fusion power, but also in fundamental understanding of 
the underlying physics. Nonetheless, many important questions, especially in the interaction between transport, MHD stability, and energetic particle physics, remain in the quest to develop fusion energy. Many of these will be answered in existing devices or in devices operating in deuterium; however, some will only be answered on a burning plasma device operating near ignition.

The work on TFTR was the product of a great team effort by scientists, engineers and technical staff from PPPL, and other national and international laboratories, universities and industries. The work highlighted here is but a short and incomplete discussion of a large body of work. We are grateful to R. Davidson and R. Goldston for their steadfast support and encouragement. This work was performed under U.S. DOE Contract No. DE-AC02-76CH03073.

\section{References}

Aymar, R., et al., 1997, in Fusion Energy: Proceedings of the Sixteenth IAEA Fusion Energy Conference, Montreal, 1996, (IAEA, Vienna), Vol. 1, p. 3.

Bathke, C.G., and the ARIES Research Team, 1994, Fusion Technol., 26, 1163.

Bell, R. E., F. M. Levinton, S. H. Batha, E. J. Synakowski, and M. C. Zarnstorff, 1998, Plasma Phys. Control. Fusion 40, 609.

Bell, M.G., et al., 1989, in Plasma Phys. Control. Nuclear Fusion Research: Proceedings of the Twelfth International Conference Nice, 1989 (IAEA, Vienna), I, 27.

Bell, M. G., et al., 1998, presented at 25th EPS Conference on Controlled Fusion and Plasma Physics to be published in Plasma Physics and Controlled Fusion.

Berk, H.L., et al., 1997, in Fusion Energy: in Proceedings of the Sixteenth IAEA Fusion Energy Conference, Montreal, 1996, (IAEA, Vienna), Vol. 2, p. 439. 
Bessenrodt-Weberpals, M., et al., 1993, Nucl. Fus. 33, 1205.

Bickerton, R.J., et al., 1971, Nature Phys. Sci. 224, 110.

Budny, R.V., 1994, Nucl. Fusion 34, 1247.

Budny R.V., et al., 1994, in Proceedings of the 21st EPS Conference on Controlled Fusion and Plasma Physics, Montpellier, edited by E. Joffrin, P. Platz, P.E. Stott (EPS, Geneva) 1, 82.

Burrell, K. H., 1997, Phys. of Plasma, 4, 1499.

Chang, Z., J.D. Callen, E.D. Fredrickson, R.V. Budny, C.C. Hegna, K.M. McGuire, and M. Zarnstorff, 1995, Phys. Rev. Lett. 74, 4663.

Chang, Z., et al., 1997, Phys. Plasmas 4, 1610.

Cheng, C.Z., G.Y. Fu, and J.W. Van Dam, 1989, Theory of Fusion Plasmas (Proc. Joint Varenna - Lausanne Int. Workshop, Chexbres, Switzerland, 1988), Editrice Composition, Bologna, 259.

Darrow, D.S., 1997, Nucl. Fusion 37, 939.

Darrow, D.S., et al., 1996, Phys. Plasmas 3, 1875

Ernst, D. R., 1997, Ph. D. thesis, Physics Dept., Massachusetts Institute of Technology.

Ernst, D. R., 1998a, Phys. Plasmas, 5, 565.

Ernst, D. R. 1998b, "The Unifying Role of Radial Electric Field Shear in the Confinement Trends of TFTR Supershot Plasmas" submitted to Phys. Rev. Lett. 
Evensen, H. R. Fonck, S. Paul, G. Rewoldt, S. Scott, W. Tang, and M. Zarnstorff, 1998, Nucl. Fusion 38, 237.

Fisch, N.J., and J.-M. Rax, 1992, Phys. Rev. Lett. 69, 612.

Fisch, N. J. and M. C. Herrmann, 1998, presented at 25th EPS Conference on Controlled Fusion and Plasma Physics to be published in Plasma Physics and Controlled Fusion.

Fisher, R.K., J.M. McChesney, P.B. Parks, H.H. Duong, S.S. Medley, A.L. Roquemore, D.K. Mansfield, R.V. Budny, M.P. Petrov, and R.E. Olson, 1995, Phys. Rev. Lett. 75, 846.

Fonck, R. J., et al. 1993, Phys. Rev. Lett. 70, 3736.

Fredrickson, E.D., K.M. McGuire, Z.Y. Chang, A. Janos, J. Manickam, and G. Taylor, 1996, Phys. Plasmas 3, 2620.

Fu, G.Y., et al., 1996, Phys. Plasmas 3, 4036.

Fu, G.Y., et al., 1997, in Nuclear Fusion: Proceedings of the Sixteenth IAEA Fusion Energy Conference, Montreal, 1996, (IAEA Vienna), Vol. 2, p. 453 .

Fujita, T., et al., 1997, Phys. Rev. Lett., 78, 2377.

Galeev, A.A., 1970, Zh. Eksp. Teor. Fiz. 59, 1378 (Sov. Phys. - JETP) 32, 752

Gibson, A. and the JET Team, Phys. Plasmas 5, 1839.

Gryaznevich, M. et al., 1998, Phys. Rev. Lett., 80, 3972.

Hawryluk, R. J. 1998, Rev. Mod. Phys. 70, 537.

Hawryluk, R.J., et al., 1994, Phys. Rev. Lett. 72, 3530.

Hawryluk, R. J. et al., 1998, Phys. Plasmas 5, 1577. 
Heidbrink, W.W., and G.J. Sadler, 1994, Nucl. Fusion 34, 535.

Herrmann, M.C., 1998, Ph. D. Thesis, Princeton University.

Herrmann, M.C., and N.J. Fisch, 1997, Phys. Rev. Lett. 79, 1495.

Hsu, C.T., C.Z. Cheng, P. Helander, D.J. Sigmar, and R. White, 1994, Phys. Rev. Lett. 72, 2503.

JET Team, 1992, Nucl. Fusion 32, 187.

Kessel, C., et al., 1994, Phys. Rev. Lett. 72, 1212.

Kinsey, Jon E., and G. Bateman, 1996, Phys. Plasmas, 3, 3344.

Kotschenreuther, M., W. Dorland, M. Beer, and G. Hammett, 1995, Phys. Plasmas 2, 2381.

Levinton, F.M., L. Zakharov, S.H. Batha, J. Manickam, and M.C. Zarnstorff, 1994, Phys. Rev. Lett. 72, 2895.

Levinton, F.M., et al., 1995, Phys. Rev. Lett. 75, 4417.

Levinton, F. M., R. E. Bell, E. J. Synakowski, and M. C. Zarnstorff, 1998, Phys. Rev. Lett. 80, 4887.

Lin, Z., W. Tang and W. W. Lee, 1997, Phys. Rev. Lett. 78, 456.

Manfredi G. and R.O. Dendy, 1996, Phys. Rev. Lett. 76, 4360.

Manickam, J., et al., 1997, in Fusion Energy: Proceedings of the Sixteenth IAEA Fusion Energy Conference, Montreal, 1996, (IAEA, Vienna), Vol. 1, p. 453.

Mansfield, D.K., et al., 1996, Phys. Plasmas 3, 1892. 
Mazzucato, E. and R. Nazikian, 1993, Phys. Rev. Lett. 71, 1840.

Mazzucato, E., et al., 1996, Phys. Rev. Lett. 77, 3145.

McKee, G.R., et al., 1995, Phys. Rev. Lett. 75, 649.

McKee, G.R., et al., 1997, Nucl. Fusion 37, 501.

Meade, D.M., et al., 1991, in Plasma Physics and Controlled Nuclear Fusion Research: Thirteenth Conference Proceedings, Washington, US (IAEA, Vienna) 1, 9.

Medley, S. S., et al., 1998, "Confined Trapped - Alpha Behaviour in TFTR Deutrium-Tritium Plasma” to be published in Nucl. Fusion.

Murakami, M. et al. 1986, Plasma Phys. Controlled Fusion 28, 17.

Mynick, H.E., and R.E. Duvall, 1989, Phys. Fluids B, 1, 750.

Mynick, H.E., and N. Pomphrey, 1994, Nucl. Fusion 34, 1277.

Myra, J.R. et al., 1993, Phys. Fluids B, 5, 1160.

Nagayama, Y., et al., 1993, Phys. Fluids B5, 2571.

Nazikian, R., et al., 1997, Phys. Rev. Lett. 78, 2976.

Ono, M., 1993, Phys. Fluids B 5, 241.

Ongena, J., M. Evrard, and D. McCune, 1998, Trans. Fusion Tech., 33, 181.

Ozeki, T., et al., 1993, in Plasma Physics and Controlled Fusion Research: Proceedings of the Fourteenth International Conference, Würzburg, Germany (IAEA Vienna) 2, 187. 
Park, W., E.D. Fredrickson, A. Janos, J. Manickam, and W.T. Tang, 1995, Phys. Rev. Lett. 75, 1763.

Reiman, A., R. Goldston, L. Ku, D. Monticello, H. Mynick, G. Neilson, M. Zarnstorff, I. Zatz, W. Cooper and A. Boozer, 1998, J. Plasma Fusion Res. 1, 429.

Sabbagh, S.A., et al., 1995, in Tokamak Concept Improvement, Proceedings of the Workshop held at the 1994 International School of Plasma Physics,Varenna, Italy, edited by S. Bernabei, N. Sauthoff, and E. Sindoni (Editrice Compositori, Bologna, Italy), p. 219.

Scott, S., et al., 1990a, Phys. Rev. Lett. 64, 531

Scott, S., et al., 1990b, Phys. Fluids B 2, 1300.

Scott, S.D., et al., 1995, Phys. Scr. 51, 394.

Scott, S.D., et al., 1997, in Fusion Energy: Proceedings of the Sixteenth IAEA Fusion Energy Conference, Montreal, 1996, (IAEA, Vienna) Vol. 1, p. 573.

Skinner, C., J. Hosea, D. Mueller, P. Coad, G. Frederici and G. Haange, 1998a "Tritium Experience in Large Tokamaks: Application to ITER", PPPL-3000, submitted to Nucl. Fusion.

Skinner, C., et al., 1998b, in the Proceedings of the Symposium on Fusion Technology, Marseille, 7-11 September.

Spong, D.A., C.L. Hedrick, and B.A. Carreras, 1995, Nucl. Fusion 35, 1687.

Strachan, J.D., et al., 1987, Phys. Rev. Lett. 58, 1004.

Strachan, J.D., et al., 1994, Phys. Rev. Lett. 72, 3526.

Strait, E. J., et al., 1995, Phys. Rev. Lett. 75, 4421. 
Sykes, A., M. F. Turner, and S. Patel, 1983, in Proc. of the Eleventh European Conference on Controlled Fusion and Plasma Physics Acheivement 7d, 373.

Synakowski, E.J., et al., 1993, Phys. Fluids, B, 5, 2215.

Synakowski, E.J., et al., 1995, Phys. Rev. Lett. 75, 3689.

Synakowski, E.J., et al., 1997, Phys. Rev. Lett. 78, 2972.

Taylor, G., et al., 1996, Phys. Rev. Lett. 76, 2722.

Thomas, P. R. et al., 1998, Phys. Rev. Lett. 80, 5548.

Troyon, F., R. Gruber, H. Saurenmann, S. Semenzato, and S. Succi, 1984, Plasma Phys. Control. Fusion 26, 209.

White, R.B., Y. Wu, Y. Chen, E.D. Fredrickson, D.S. Darrow, M.C. Zarnstorff, J.R. Wilson, S.J. Zweben, K.W. Hill, and G.Y. Fu, 1995, Nucl. Fusion 35, 1707.

Wilson, J. R., et al., 1998, Phys. Plasmas 5, 1721.

Zarnstorff, M.C., et al., 1988, Phys. Rev. Lett. 60, 1306.

Zweben, S.J., et al., 1997, Plasma Phys. Control. Fusion 39, A275. 


\section{Appendix: TFTR Group:}

S. Batha, ${ }^{1}$ W. Blanchard, M. Beer, M.G. Bell, R.E. Bell, H. Berk,${ }^{2}$ S. Bernabei, M. Bitter, B. Breizman, ${ }^{2}$ N.L. Bretz, R. Budny, C.E. Bush, ${ }^{3}$ J. Callen, ${ }^{4}$ R. Camp, S. Cauffman, Z. Chang, C.Z. Cheng, D.S. Darrow, R.O. Dendy, ${ }^{5}$ W. Dorland ${ }^{2}$ H. Duong, ${ }^{6}$ P.C. Efthimion, D. Ernst, ${ }^{7}$ N.J. Fisch, R. Fisher, ${ }^{5}$ R.J. Fonck, ${ }^{4}$ E.D. Fredrickson, G.Y. Fu, H.P. Furth, N.N. Gorelenkov, ${ }^{8}$ B. Grek, L.R. Grisham, G.W. Hammett, G.R. Hanson, ${ }^{3}$. R. J. Hawryluk, H.W. Herrmann, M.C. Herrmann, ${ }^{5}$ K.W. Hill, J. Hogan, ${ }^{3}$ J.C. Hosea, W.A. Houlberg, ${ }^{3}$ M. Hughes, ${ }^{9}$ R.A. Hulse, D.L. Jassby, F.C. Jobes, D.W. Johnson, R. Kaita, S. Kaye, J.S. Kim ${ }^{4}$ M. Kissick,${ }^{4}$ A.V. Krasilnikov, ${ }^{8}$ H. Kugel, A. Kumar, ${ }^{10}$ B. Leblanc, F.M. Levinton, ${ }^{1}$ C. Ludescher, R.P. Majeski, J. Manickam, D.K. Mansfield, E. Mazzucato, J. McChesney, ${ }^{7}$ D.C. McCune, K.M. McGuire, D.M. Meade, S.S. Medley, R. Mika, D.R. Mikkelsen, S.V. Mirnov, ${ }^{8}$ D. Mueller, A. Nagy, G.A. Navratil,${ }^{11}$ R. Nazikian, M. Okabayashi, H.K. Park, W. Park, S.F. Paul, G. Pearson, M.P. Petrov, ${ }^{12}$ C.K. Phillips, M. Phillips, ${ }^{9}$ A.T. Ramsey, M.H. Redi, G. Rewoldt, S. Reznik, ${ }^{15}$ A.L. Roquemore, J. Rogers, E. Ruskov, S.A. Sabbagh, ${ }^{11}$ M. Sasao ${ }^{13}$ G. Schilling, J. Schivell, G.L. Schmidt, S.D. Scott, I. Semenov, ${ }^{8}$ C.H. Skinner, T. Stevenson, B.C. Stratton, J.D. Strachan, W. Stodiek, E. Synakowski, H. Takahashi, W. Tang, G. Taylor, M.E. Thompson, S. Von Goeler, A. Von Halle, R.T. Walters, R. White, R.M. Wieland, M. Williams, J.R. Wilson, K.L. Wong, G.A. Wurden, ${ }^{14}$ M. Yamada, V. Yavorski ${ }^{15}$ K.M. Young, L. Zakharov, M.C. Zarnstorff, S.J. Zweben

1. Permanent Address: Fusion Physics and Technology, Torrance, California

2. Permanent Address: University of Texas, Institute for Fusion Studies, Austin, Texas

3. Permanent Address: Oak Ridge National Laboratory, Oak Ridge, Tennessee

4. Permanent Address: University of Wisconsin, Madison Wisconsin

5. Permanent Address: Culham Laboratory, Abingdon, Oxford, United Kingdom

6. Permanent Address: General Atomics, San Diego, California

7. Permanent Address: Massachusetts Institute of Technology, Cambridge, Massachusetts

8. Permanent Address: Troitsk Institute of Innovative and Thermonuclear Research, Moscow, Russia

9. Permanent Address: Northrop-Grumman Corporation, Princeton, New Jersey

10. Permanent Address: University of California, Los Angeles, California

11. Permanent Address: Columbia University, New York, New York

12. Permanent Address: Ioffe Physical-Technical Institute, St. Petersburg, Russia

13. Permanent Address: National Institute for Fusion Science, Nagoya, Japan

14. Permanent Address: Los Alamos National Laboratory, Los Alamos, NM

15. Permanent Address: Ukrainian Institute of Nuclear Research, Kiev, Ukraine 
Table I. Summary of TFTR Experimental Parameters Achieved in High Performance $\underline{\underline{\text { Supershot Discharges }}}$

\begin{tabular}{|c|c|c|c|c|c|}
\hline Parameters & Units & 68522 & 76778 & 80539 & 83546 \\
\hline Plasma Current $\left(\mathrm{I}_{\mathrm{p}}\right)$ & MA & 2.0 & 2.5 & 2.7 & 2.3 \\
\hline Toroidal Field $\left(\mathrm{B}_{\mathrm{T}}\right)$ & $\mathrm{T}$ & 5.0 & 5.1 & 5.6 & 5.5 \\
\hline NB Power $\left(\mathrm{P}_{\mathrm{b}}\right)$ & MW & 30.8 & 33.7 & 39.6 & 17.4 \\
\hline Tritium NB Power & MW & 0 & 20.0 & 25.5 & 17.4 \\
\hline Central Electron Density $\left[n_{\mathrm{e}}(0)\right]$ & $10^{19} \mathrm{~m}^{-3}$ & 9.6 & 8.5 & 10.2 & 8.5 \\
\hline $\begin{array}{l}\text { Central Hydrogenic Density } \\
\end{array}$ & $10^{19} \mathrm{~m}^{-3}$ & 6.8 & 6.3 & 6.7 & 6.6 \\
\hline 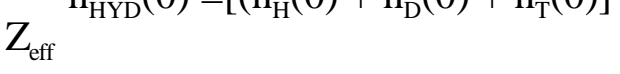 & & 2.6 & 2.2 & 2.4 & 2.0 \\
\hline $\mathrm{T}_{\mathrm{e}}(0)$ & $\mathrm{keV}$ & 11.7 & 11.5 & 13 & 12.0 \\
\hline $\mathrm{T}_{\mathrm{i}}(0)$ & $\mathrm{keV}$ & 29.0 & 44 & 36 & 43 \\
\hline Plasma Energy $\left(\mathrm{W}_{\mathrm{TOT}}\right)$ & MJ & 5.4 & 6.5 & 6.9 & 4.9 \\
\hline $\mathrm{dW}_{\text {тот }} / \mathrm{dt}$ & MW & 2.1 & 7.5 & 0 & 3.0 \\
\hline$\tau_{\mathrm{E}}=\mathrm{W}_{\mathrm{TOI}} /\left(\mathrm{P}_{\mathrm{TOT}}-\mathrm{dW} / \mathrm{dt}\right)$ & $\mathrm{S}$ & 0.19 & 0.24 & 0.18 & 0.34 \\
\hline $\mathrm{n}_{\mathrm{HYD}}(0) \mathrm{T}_{\mathrm{i}}(0) \tau_{\mathrm{E}}$ & $\begin{array}{c}10^{20} \mathrm{~m}^{-3} \\
\mathrm{keVs}\end{array}$ & 3.9 & 7.1 & 4.3 & 9.6 \\
\hline $\mathrm{n}_{\mathrm{HYD}}(0) \mathrm{T}_{\mathrm{i}}(0) \tau_{\mathrm{E}}^{*}$ & $\begin{array}{c}10^{20} \mathrm{~m}^{-3} \\
\mathrm{keVs}\end{array}$ & 3.6 & 5.5 & 4.2 & 8.0 \\
\hline Ratio of average $\mathrm{T}$ to $(\mathrm{D}+\mathrm{T})$ density & & 0 & 0.5 & 0.47 & 0.58 \\
\hline Maximum Fusion Power & MW & 0.065 & 9.3 & 10.7 & 2.8 \\
\hline$\beta_{\mathrm{N}}$ & & 2.1 & 2.0 & 1.8 & 1.5 \\
\hline$\beta_{\mathrm{N}} *$ & & 3.5 & 3.1 & 3.0 & 3.0 \\
\hline
\end{tabular}


Figure Captions

Fig. 1 Comparison of temperature and density profiles before (dashed curve) and after (solid curve) the transition in an enhanced reverse-shear discharge. Note the strong ion temperature and density gradients. (Levinton et al. 1995)

Fig. 2 In enhanced reverse-shear discharges: (a) the inferred ion thermal diffusivity and (b) particle diffusivity are observed to decrease dramatically (Levinton et al. 1995). For comparison, the standard calculation of neoclassical ion heat conductivity is shown, as well, as the calculations by Lin et al. (1997).

Fig. 3 Evolution of the central electron density, ion temperature, electron temperature, stored energy, and fusion power for TFTR shot No. 80539 (Hawryluk 1998).

Fig. 4 Absolute intensity measurement of the charge exchange recombination signal for low energy alpha particle $\mathrm{E}_{\alpha}=0.15$ $0.6 \mathrm{MeV}$ as a function of minor radius are in good agreement with TRANSP calculations indicating good radial confinement. (McKee et al. 1997)

Fig. 5. Comparison of the electron temperature profile for a set of similar D-T and D discharges with comparable energy confinement times and neutral-beam heating power. The measured difference in electron temperature is consistent with the predicted change due to alpha heating. (Taylor et al. 1996)

Fig. 6 Alpha-driven TAE mode in TFTR occurring $\approx 0.1 \mathrm{~s}$ after neutral beam injection in a D-T discharge with weak central magnetic shear. a) The evolution of $\beta_{\mathrm{N}}$ and $\beta_{\alpha}$. b) The occurrence of magnetic fluctuations with different toroidal mode numbers is observed. c) The evolution of the frequency 
of the magnetic fluctuations. The frequency is consistent with the density-dependent TAE frequency, and mode timing is in reasonable agreement with the theoretical prediction based on beam ion damping (Nazikian et al. 1997).

Fig. $7 \quad$ Illustration of the strong coupling in high $\beta$ discharges between the pressure and density gradients, the q-profile and the shear flow. In contrast with present experiments, in which the externally applied heating, torque and current drive play a major role in determining the operating point, in an ignited plasma they are small compared with the internal processes. 

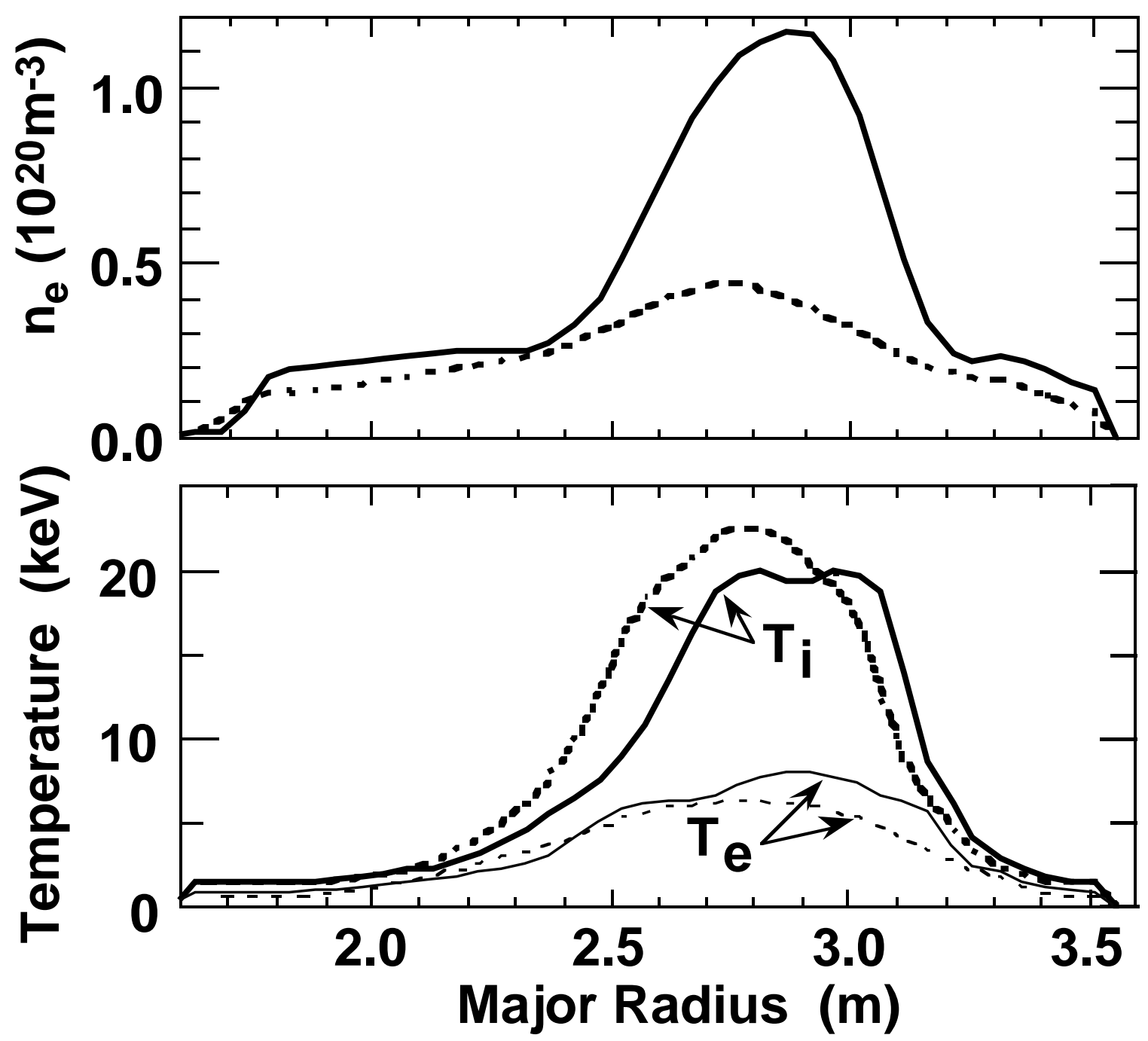

Fig. 1 Comparison of temperature and density profiles before (dashed curve) after (solid curve) the transition in an enhanced reverse-shear discharge. Note the strong ion temperature and density gradients. (Levinton et al. 1995) 

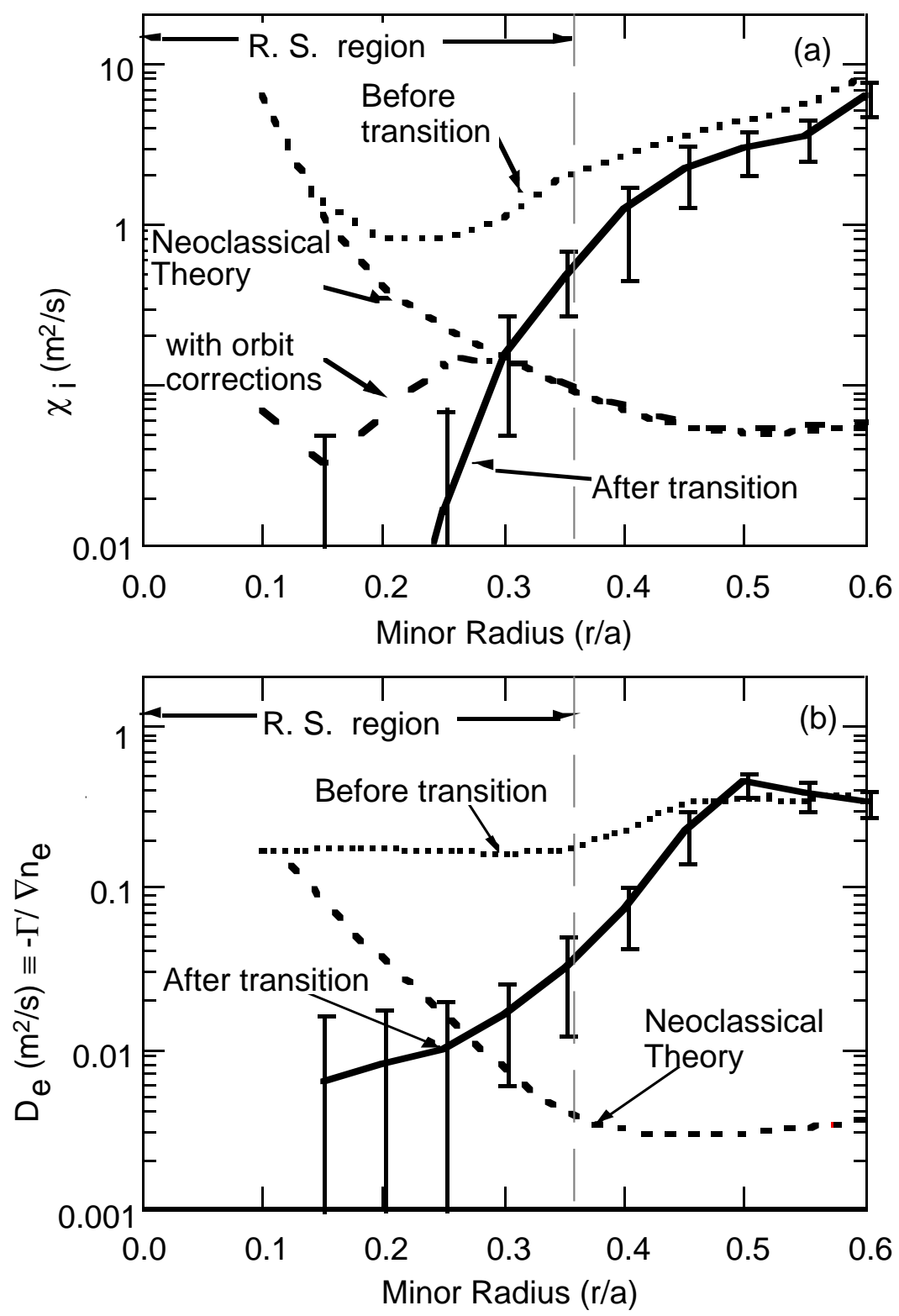

Fig. 2 In enhanced reverse-shear discharges: (a) the inferred ion thermal diffusivity and (b) particle diffusivity are observed to decrease dramatically (Levinton et al. 1995). For comparison, the standard calculation of neoclassical ion heat conductivity is shown, as well, as the calculations by Lin et al. (1997). 


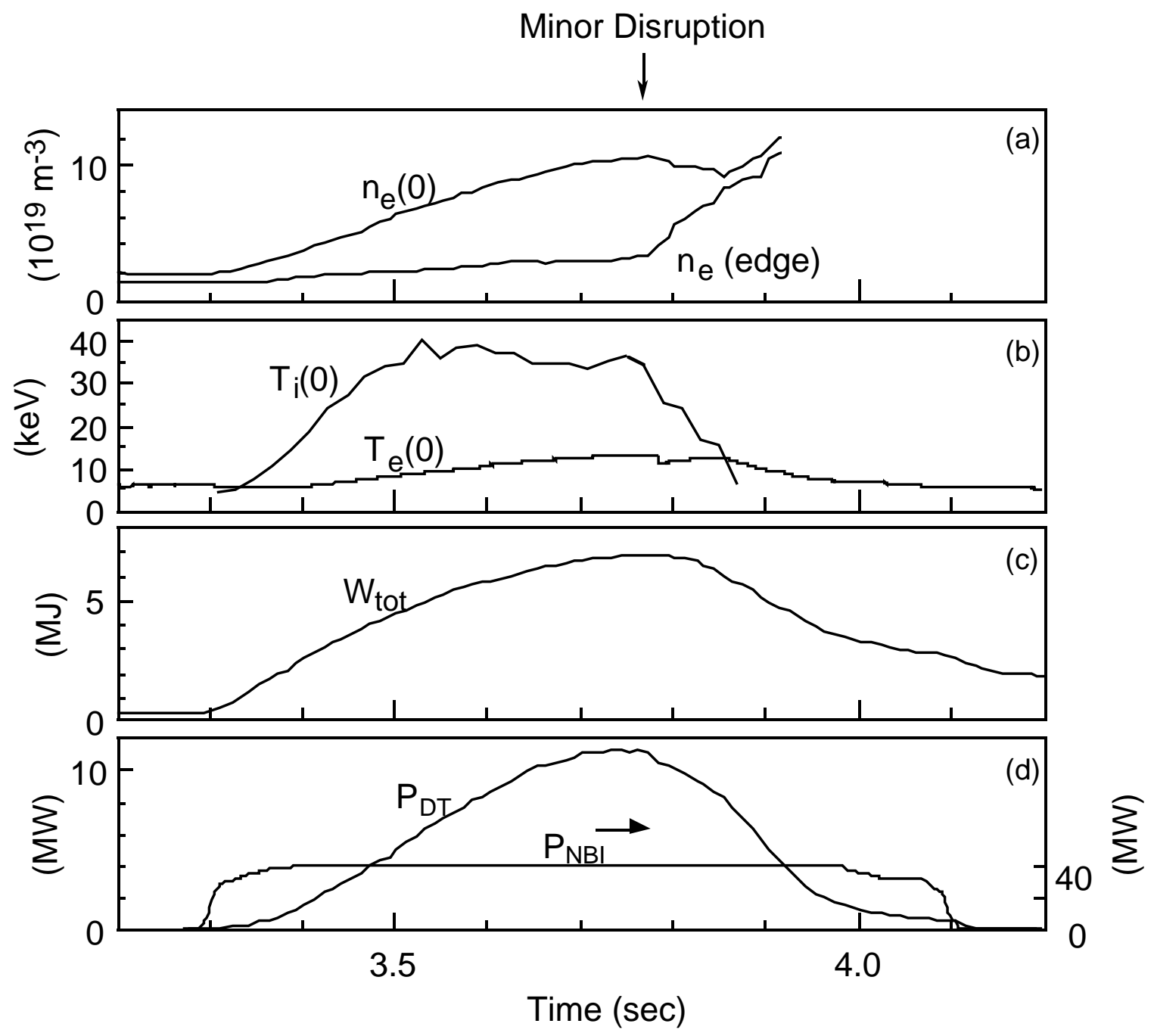

Fig. 3 Evolution of the central electron density, ion temperature, electron temperature, stored energy, and fusion power for TFTR shot No. 80539 (Hawryluk 1998). 


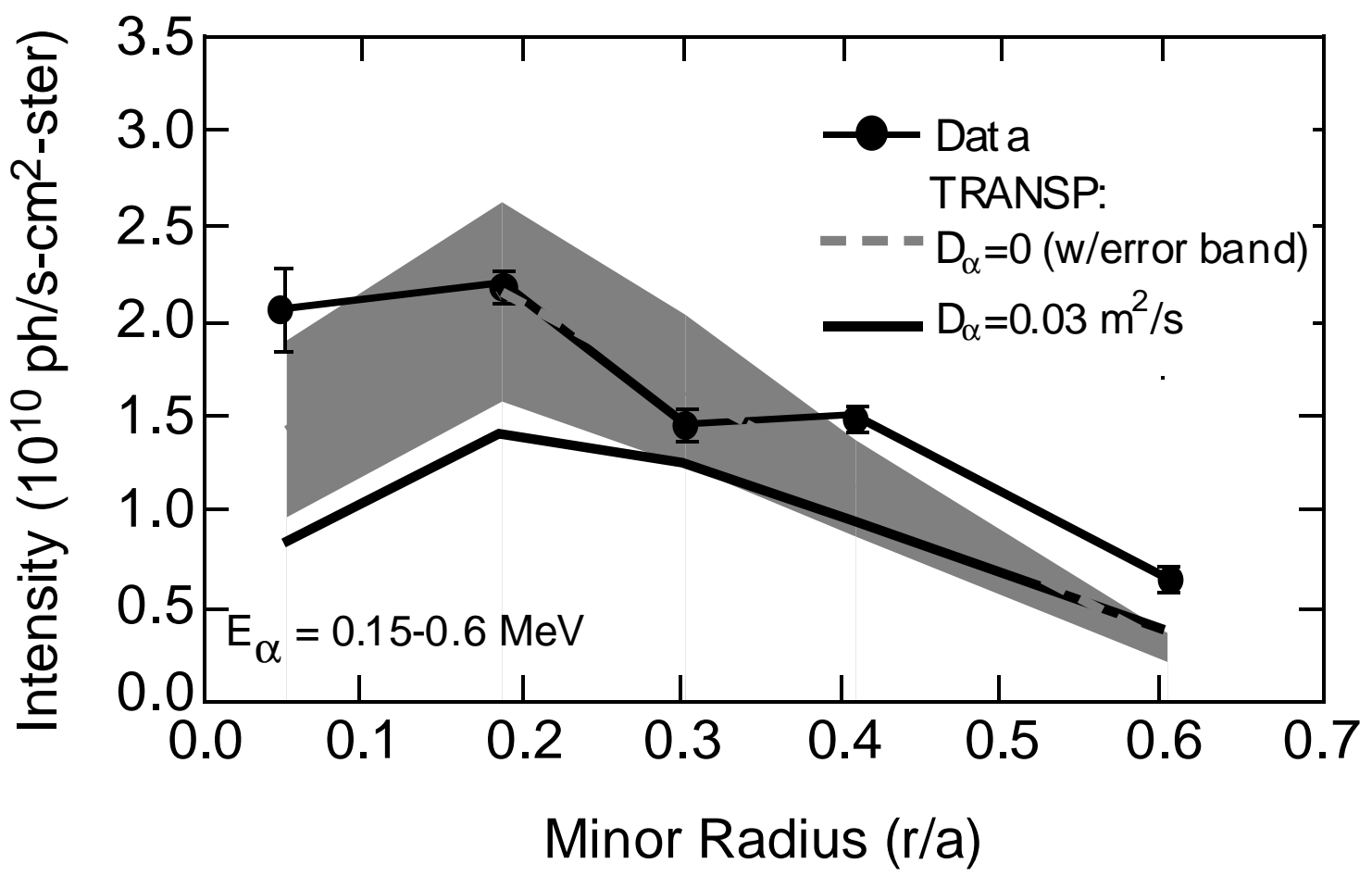

Fig. 4 Absolute intensity measurement of the charge exchange recombination signal for low energy alpha particle $E_{\alpha}=0.15$ $0.6 \mathrm{MeV}$ as a function of minor radius are in good agreement with TRANSP calculations indicating good radial confinement. (McKee et al. 1997) 


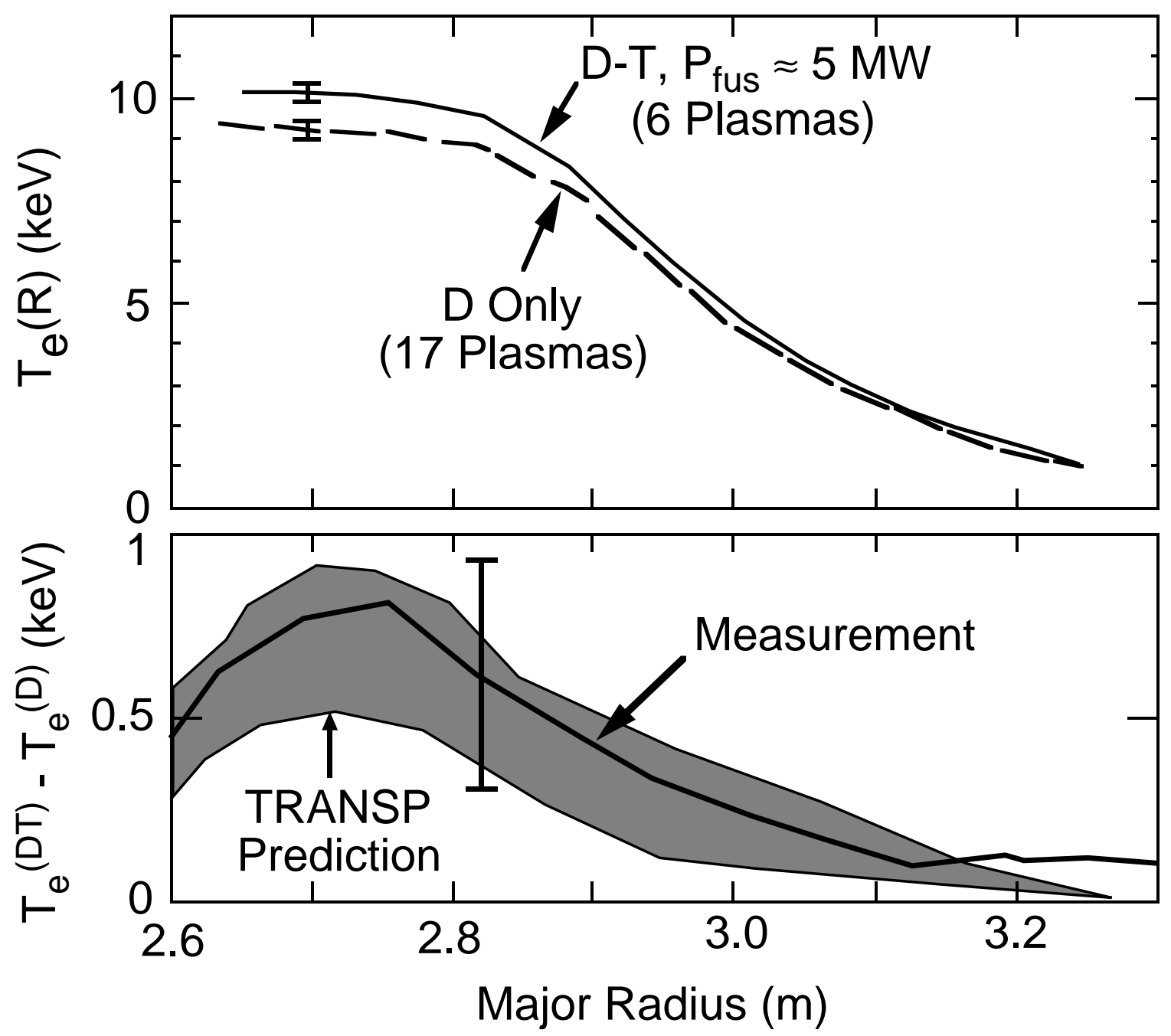

Fig. 5. Comparison of the electron temperature profile for a set of similar D-T and D discharges with comparable energy confinement times and neutral-beam heating power. The measured difference in electron temperature is consistent with the predicted change due to alpha heating. (Taylor et al. 1996) 


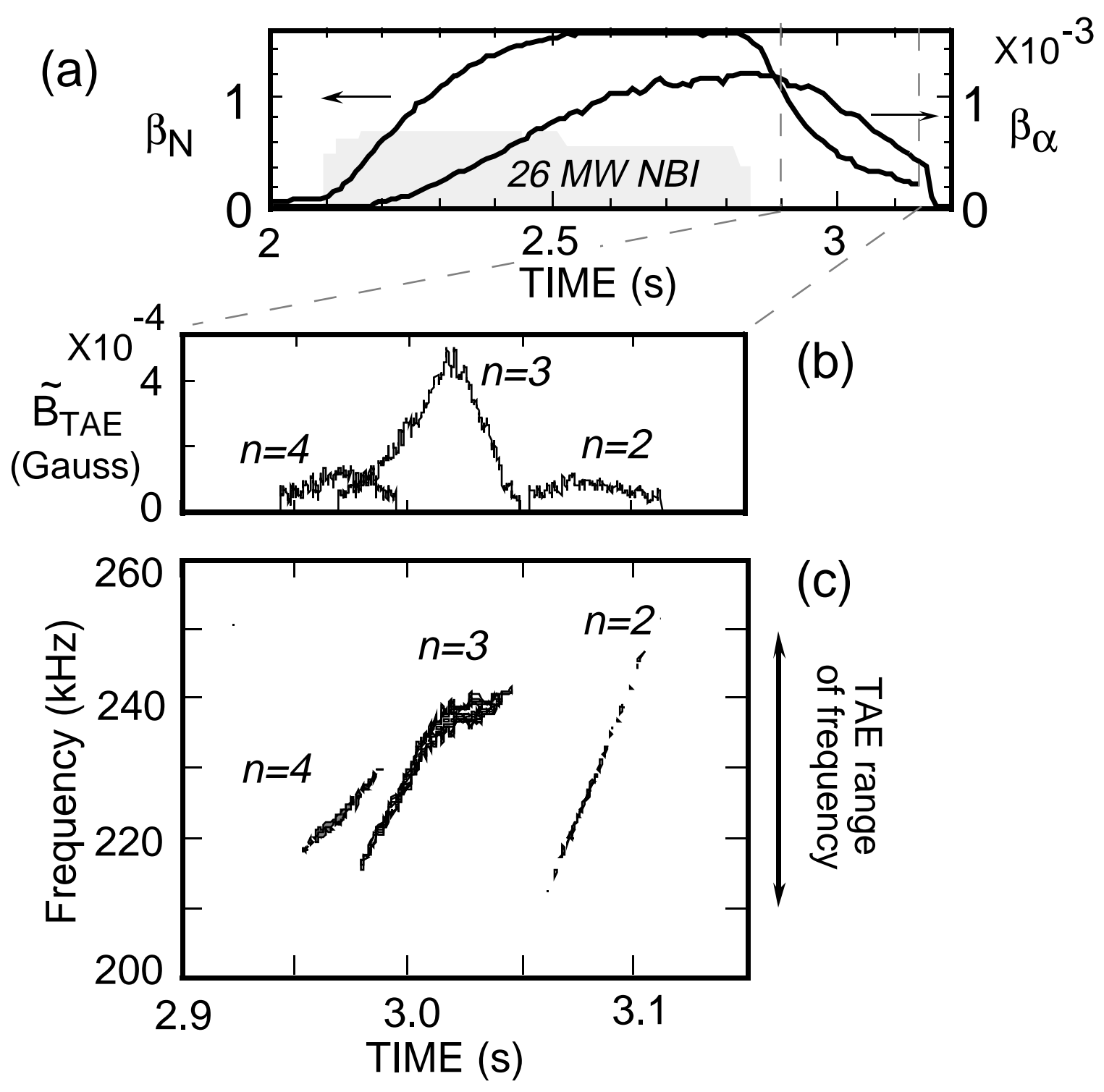

Fig. 6 Alpha-driven TAE mode in TFTR occurring $\approx 0.1 \mathrm{~s}$ after neutral beam injection in a D-T discharge with weak central magnetic shear. a) The evolution of $\beta_{\mathrm{N}}$ and $\beta_{\alpha}$. b) The occurrence of magnetic fluctuations with different toroidal mode numbers is observed. c) The evolution of the frequency of the magnetic fluctuations. The frequency is consistent with the density-dependent TAE frequency, and mode timing is in reasonable agreement with the theoretical prediction based on beam ion damping (Nazikian et al. 1997). 


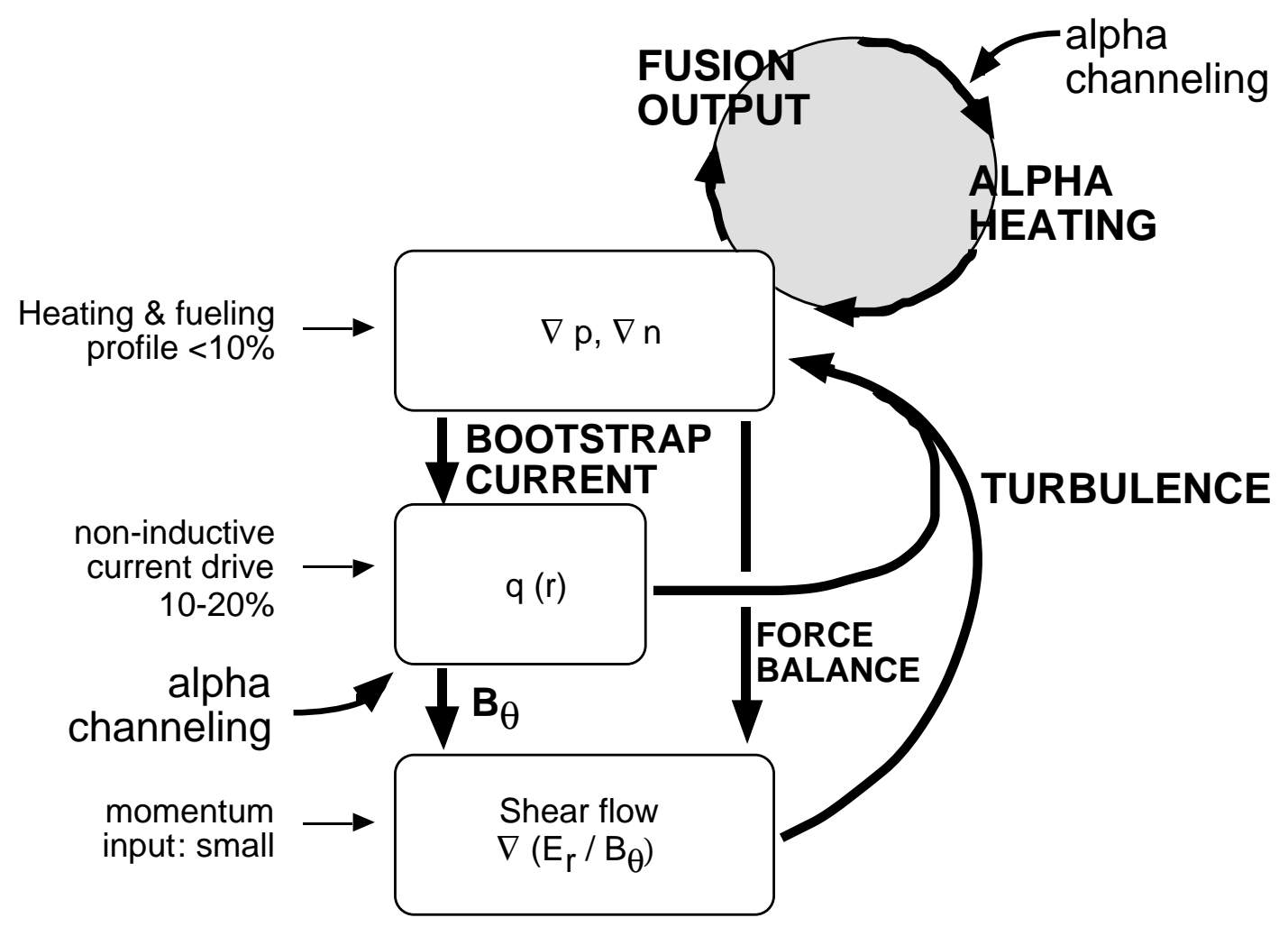

Fig. $7 \quad$ Illustration of the strong coupling in high $\beta$ discharges between the pressure and density gradients, the q-profile and the shear flow. In contrast with present experiments, in which the externally applied heating, torque and current drive play a major role in determining the operating point, in an ignited plasma they are small compared with the internal processes. 